\title{
GENERATING SERIES IN THE COHOMOLOGY OF HILBERT SCHEMES OF POINTS ON SURFACES
}

\author{
SAMUEL BOISSIÈRE AND MARC A. NIEPER-WISSKIRCHEN
}

\begin{abstract}
In the study of the rational cohomology of Hilbert schemes of points on a smooth surface, it is particularly interesting to understand the characteristic classes of the tautological bundles and the tangent bundle. In this note we pursue this study. We first collect all results appearing separately in the literature and prove some new formulas using Ohmoto's results on orbifold Chern classes on Hilbert schemes. We also explain the algorithmic counterpart of the topic: the cohomology space is governed by a vertex algebra that can be used to compute characteristic classes. We present an implementation of the vertex operators in the rewriting logic system MAUDE, and address observations and conjectures obtained after symbolic computations.
\end{abstract}

\section{PART I. PRELIMINARIES}

\section{Introduction}

Let $S$ be a smooth quasi-projective complex surface and $n \geqslant 0$ an integer. The Hilbert scheme of $n$ points on $S$, denoted by $S^{[n]}$, is the moduli space of generalized $n$-tuples on $S$, that is, zero-dimensional subschemes of length $n$ on $S . S^{[n]}$ is smooth of complex dimension $2 n$. When working in the rational cohomology of Hilbert schemes, it is usual to consider the total Hilbert scheme:

$$
\operatorname{Hilb}(S):=\coprod_{n \geqslant 0} S^{[n]}
$$

whose total cohomology space is:

$$
\mathbb{H}_{S}:=\prod_{n \geqslant 0} \bigoplus_{i=0}^{4 n} H^{i}\left(S^{[n]}\right) .
$$

The space $\mathbb{H}_{S}$ is completed bigraded by conformal weight $n$ and cohomological degree $i$.

Consider the tangent bundle $T_{S}^{n}$ on $S^{[n]}$ of rank $2 n$. Any characteristic class $\phi$ (such as the Chern class, the Segre class, the Todd class or the Chern character) 
can be applied to this bundle and gives, after summing over all values of $n$, an element:

$$
\Phi(S):=\sum_{n \geqslant 0} \phi\left(T_{S}^{n}\right) \in \mathbb{H}_{S} .
$$

There exists a universal way of presenting these characteristic classes, involving the canonical class $K_{S}$ and the Euler class $e_{S}$ of $S$, depending on universal constants independent of $S$, indexed by partitions of integers.

Similarly, any vector bundle $F$ of rank $r$ on $S$ defines in a tautological way a vector bundle $F^{[n]}$ of rank $n \cdot r$ on $S^{[n]}$, called a tautological bundle. Any characteristic class $\phi$ can be applied to these bundles and gives an element:

$$
\Phi(F):=\sum_{n \geqslant 0} \phi\left(F^{[n]}\right) \in \mathbb{H}_{S} .
$$

As before, there exists a universal way of presenting these characteristic classes, involving the corresponding characteristic class $\phi(F)$, the canonical class $K_{S}$ and the Euler class $e_{S}$ of $S$, depending on universal coefficients independent of $F$ and $S$, indexed by partitions of integers.

In this paper, we are interested in the effective computation of these universal coefficients in both situations. Since the constants are independent of the surface, one may evaluate each formula on well-chosen surfaces and use appropriate tools to compute new coefficients step by step. This is one of the main tricks used to obtain the values of some series of coefficients, together with manipulations of vertex algebra operators. When these methods fail, one can make use of a suitable computer program to get information on the missing values.

In Section 2 we recall some basics on the vertex algebra structure of the total cohomology space $\mathbb{H}_{S}$ : natural vertex operators and commutation relations between them lead to effective algorithms to compute classes in $\mathbb{H}_{S}$. Part II is devoted to the case of the tautological bundles and Part III to the tangent bundle. We explain the general shape of the formulas, then present the current state of knowledge concerning the universal constants and prove some new results, and finally we produce new values obtained by an implementation of the vertex algebra structure with MAUDE [4]: the vertex operators are directly defined with their commutation rules, making the program clear to understand from the mathematical point of view (Part IV). To our knowledge, MAUDE has not previously been used for huge algebraic computations and simplifications. The nature of MAUDE as a rewriting system will make it easy to prove the correctness of the implemented algorithms.

\section{Notation}

We recall here some notation and classical constructions. For further details, refer to $[\mathbf{1}, \mathbf{3}, \mathbf{6}, \mathbf{7}]$. 


\subsection{Combinatorics}

Let $\mathcal{P}$ be the set of all partitions of integers. A partition $\lambda=\left(\lambda_{1}, \lambda_{2}, \ldots\right)$ is likewise denoted by $\lambda=\left(1^{m_{1}}, 2^{m_{2}}, \ldots\right)$, and we define:

$$
\begin{array}{rlrl}
\ell(\lambda) & :=\sum_{i \geqslant 1} m_{i} & \text { (the length); } & \|\lambda\|:=\sum_{i \geqslant 1} i^{2} m_{i} ; \\
|\lambda|:=\sum_{i \geqslant 1} i m_{i} & \text { (the weight); } & \lambda !:=\prod_{i \geqslant 1} m_{i} !
\end{array}
$$

We often write $n$ instead of $(n)=\left(n^{1}\right)$.

\section{2. $\quad$ Vertex operators}

A linear operator $\mathfrak{f} \in \operatorname{End}\left(\mathbb{H}_{S}\right)$ is homogeneous of conformal weight $u$ and cohomological degree $v$ if for any $n$ one has $\mathfrak{f}\left(H^{i}\left(S^{[n]}\right)\right) \subset H^{i+v}\left(S^{[n+u]}\right)$. The commutator of two homogeneous operators $\mathfrak{f}, \mathfrak{g}$ is defined by:

$$
[\mathfrak{f}, \mathfrak{g}]:=\mathfrak{f} \circ \mathfrak{g}-(-1)^{|\mathfrak{f}| \cdot|\mathfrak{g}|} \mathfrak{g} \circ \mathfrak{f}
$$

where $|\cdot|$ denotes the cohomological degree.

The total cohomology space $\mathbb{H}_{S}$ is computed with the help of Nakajima's creation operators (see [9]):

$$
\mathfrak{q}_{k}: H^{*}(S) \longrightarrow \operatorname{End}\left(\mathbb{H}_{S}\right), \quad k \geqslant 1 \text {. }
$$

For $\alpha \in H^{i}(S)$, the operator $\mathfrak{q}_{k}(\alpha)$ has conformal weight $k$ and cohomological degree $2(k-1)+i$. We make use of the following abbreviation: for $k \geqslant 1$, the push-forward induced by the diagonal inclusion $\Delta^{k}: S \rightarrow S^{k}$ gives a map $\Delta_{!}^{k}: H^{*}(S) \rightarrow H^{*}(S)^{\otimes k}$. For $\Delta_{!}^{k} \alpha=\sum_{i} \alpha_{i, 1} \otimes \cdots \otimes \alpha_{i, k}$ and $\lambda=\left(\lambda_{1}, \ldots, \lambda_{k}\right)$ a partition of length $k$ we set $(\operatorname{see}[\mathbf{1}, \mathbf{3}])$ :

$$
\mathfrak{q}_{\lambda}(\alpha):=\left(\mathfrak{q}_{\lambda_{1}} \circ \cdots \circ \mathfrak{q}_{\lambda_{k}}\right) \Delta_{!}^{k}(\alpha):=\sum_{i} \mathfrak{q}_{\lambda_{1}}\left(\alpha_{i, 1}\right) \circ \cdots \circ \mathfrak{q}_{\lambda_{k}}\left(\alpha_{i, k}\right) .
$$

The unit in $H^{0}\left(S^{[0]}\right) \cong \mathbb{Q}$ is denoted $|0\rangle$, and is called the vacuum of $\mathbb{H}_{S}$. Evaluations on the vacuum, denoted by $\mathfrak{q}_{\lambda}(\alpha)|0\rangle$, provide very natural classes in $\mathbb{H}_{S}$. The unit in $\mathbb{H}_{S}$ for the cup product is given by:

$$
|1\rangle:=\sum_{n \geqslant 0} 1_{S^{(n]}}=\mathrm{e}^{\mathfrak{q}_{1}\left(1_{S}\right)}|0\rangle .
$$

\subsection{Tautological classes}

Let $\Xi_{S}^{n}$ be the universal family on $S^{[n]}$ with the following projections.

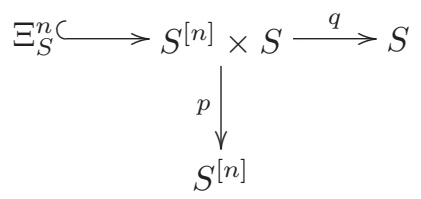

Let $F$ be a locally free sheaf on $S$. For any $n \geqslant 0$, the associated tautological bundle on $S^{[n]}$ is defined by:

$$
F^{[n]}:=p_{*}\left(\mathcal{O}_{\Xi_{S}^{n}} \otimes q^{*} F\right)
$$


It has rank $n \cdot \operatorname{rk}(F)$. This construction extends naturally to a well-defined group homomorphism:

$$
{ }^{[n]}: K(S) \rightarrow K\left(S^{[n]}\right)
$$

where $K(\cdot)$ denotes the rational Grothendieck group generated by locally free sheaves. For $u \in K(S)$, let $\mathfrak{c}(u)$ and $\mathfrak{c h}(u)$ be the linear operators acting for any $n \geqslant 0$ on $H^{*}\left(S^{[n]}\right)$ by the cup-product multiplication by the total Chern class c $\left(u^{[n]}\right)$ and the total Chern character $\operatorname{ch}\left(u^{[n]}\right)$ respectively.

In particular, taking $F=\mathcal{O}_{S}$, one defines a linear operator $\mathfrak{d} \in \operatorname{End}\left(\mathbb{H}^{S}\right)$ by:

$$
\mathfrak{d}(x):=c_{1}\left(\mathcal{O}_{S}^{[n]}\right) \cdot x, \quad \forall x \in H^{*}\left(S^{[n]}\right) .
$$

The derivative of a linear operator $\mathfrak{f} \in \operatorname{End}\left(\mathbb{H}_{S}\right)$ is defined by $\mathfrak{f}^{\prime}:=[\mathfrak{d}, \mathfrak{f}]$.

By analogy with the construction of tautological bundles, one defines a linear operation $-^{[n]}: H^{*}(S) \rightarrow H^{*}\left(S^{[n]}\right)$ : for any cohomology class $\gamma \in H^{*}(S)$ we set

$$
\gamma^{[n]}:=p_{*}\left(\operatorname{ch}\left(\mathcal{O}_{\Xi_{S}^{n}}\right) \cdot q^{*} \operatorname{td}(S) \cdot q^{*} \gamma\right)
$$

where $\operatorname{td}(S)$ denotes the Todd class of the tangent bundle on $S$ and we define an operator

$$
\mathfrak{G}(\gamma) \in \operatorname{End}\left(\mathbb{H}^{S}\right)
$$

acting on $H^{*}\left(S^{[n]}\right)$ by multiplication by $\gamma^{[n]}$.

Similarly, denoting by $-{ }^{\vee}: K(S) \rightarrow K(S)$ the natural ring involution taking the dual of a vector bundle, for $\gamma \in H^{*}(S)$ we set

$$
\left(\gamma^{[n]}\right)^{\vee}:=p_{*}\left(\operatorname{ch}\left(\mathcal{O}_{\Xi_{S}^{n}}^{\vee}\right) \cdot q^{*} \operatorname{td}(S) \cdot q^{*} \gamma\right)
$$

and we define an operator

$$
\mathfrak{G}^{\vee}(\gamma) \in \operatorname{End}\left(\mathbb{H}^{S}\right)
$$

acting on $H^{*}\left(S^{[n]}\right)$ by multiplication by $\left(\gamma^{[n]}\right)^{\vee}$.

\subsection{Cohomology of $S$}

In the rational cohomology ring of $S$, we denote the unit by $1_{S} \in H^{0}(S)$, the canonical class (that is, the first Chern class of the cotangent bundle on $S$ ) by $K_{S} \in H^{2}(S)$ and the Euler class of $S$ (that is, the second Chern class of the tangent bundle on $S$ ) by $e_{S} \in H^{4}(S)$.

\section{Part II. Characteristic Classes of tautological BUndles}

In this part, we study the characteristic classes of a tautological bundle on $S^{[n]}$ obtained from a vector bundle $F$ on $S$. We first recall the theoretic results.

\section{Shape of the formulas}

For tautological bundles, the best general result is obtained for the Chern character, as follows. 
TheOREm 3.1 (Boissière [1], Boissière \& Nieper-Wißkirchen [3]). There are unique rational constants $\alpha_{\lambda}, \beta_{\lambda}, \gamma_{\lambda}, \delta_{\lambda}$ such that for each surface $S$ and each vector bundle $F$ on $S$, the generating series of the Chern characters of the tautological bundles of $F$ is given by:

$$
\begin{aligned}
\operatorname{Ch}(F):=\operatorname{ch}(F)|1\rangle=\left(\sum_{\lambda \in \mathcal{P}}\right. & \alpha_{\lambda} \mathfrak{q}_{\lambda}(\operatorname{ch}(F))+\beta_{\lambda} \mathfrak{q}_{\lambda}\left(e_{S} \operatorname{ch}(F)\right) \\
& \left.\quad+\gamma_{\lambda} \mathfrak{q}_{\lambda}\left(K_{S} \operatorname{ch}(F)\right)+\delta_{\lambda} \mathfrak{q}_{\lambda}\left(K_{S}^{2} \operatorname{ch}(F)\right)\right)|1\rangle .
\end{aligned}
$$

For multiplicative characteristic classes, the general shape is similar. Let $\phi$ be a multiplicative characteristic class. Let $B$ the polynomial ring $\mathbb{Q}\left[r, c_{1}, c_{2}, K, e\right]$ truncated from degree 5 onwards where $\operatorname{deg}(r)=0, \operatorname{deg}\left(c_{1}\right)=2, \operatorname{deg}\left(c_{2}\right)=4$, $\operatorname{deg}(K)=2, \operatorname{deg}(e)=4$.

Theorem 3.2 (Boissière [1], Boissière \& Nieper-Wißkirchen [3]). There are unique elements $u_{\lambda}^{\phi} \in B$ such that for each surface $S$ and each vector bundle $F$ on $S$, the generating series of the $\phi$-classes of tautological bundles is given by:

$$
\Phi(F):=\sum_{n \geqslant 0} \phi\left(F^{(n]}\right)=\exp \left(\mathfrak{q}_{\lambda}\left(u_{\lambda}^{\phi}\left(\operatorname{rk}(F), c_{1}(F), c_{2}(F), K_{S}, e_{S}\right)\right)|0\rangle .\right.
$$

As we shall see in Section 5.1, the formula for the total Chern class simplifies considerably when specialized at $r=1$. As an example of the complexity in higher rank, we give in Section 5.2 the first terms of the linear series for $r \geqslant 2$.

\section{The Chern character}

In the determination of the constants in Theorem 3.1, a lot of information has already been obtained, and the result is complete for surfaces with trivial canonical class.

\subsection{The $\left(1_{S}\right)$ - and $\left(e_{S}\right)$-series}

All coefficients $\alpha_{\lambda}$ and $\beta_{\lambda}$ are known, as shown in the following formula.

Formula 4.1 (Li, Qin \& Wang [8, Corollary 4.8]). We have:

$$
\alpha_{\lambda}=\frac{(-1)^{|\lambda|-1}}{\lambda ! \cdot|\lambda| !} \quad \text { and } \quad \beta_{\lambda}=\frac{(-1)^{|\lambda|}}{\lambda !|\lambda| !} \cdot \frac{|\lambda|+\| \lambda||-2}{24} .
$$

\subsection{The $\left(K_{S}\right)$ - and $\left(K_{S}^{2}\right)$-series}

For the series concerning the canonical class, Li, Qin \& Wang [8, Corollary 4.8] write the still unknown constants $\gamma_{\lambda}$ and $\delta_{\lambda}$ as

$$
\gamma_{\lambda}=\frac{(-1)^{|\lambda|}}{\lambda ! \cdot|\lambda| !} \cdot g_{1}\left(\lambda \cup 1^{|\lambda|}\right) \quad \text { and } \quad \delta_{\lambda}=\frac{(-1)^{|\lambda|}}{\lambda !|\lambda| !} \cdot g_{2}\left(\lambda \cup 1^{|\lambda|}\right),
$$

where the functions $g_{1}, g_{2}$ depend only on the partition and $\lambda \cup 1^{|\lambda|}$ means that one adds $|\lambda|$ to the multiplicity of 1 in $\lambda$. 
In order to get information about these functions we implement the commutation relation of Lehn [6, Theorem 4.2]:

$$
\left[\mathfrak{c h}(F), \mathfrak{q}_{1}\left(1_{S}\right)\right]=\exp (\operatorname{ad} \mathfrak{d})\left(\mathfrak{q}_{1}(\operatorname{ch}(F)) .\right.
$$

This gives the following recursive formula (see $[\mathbf{1}, \S 3.3]$ ):

$$
\operatorname{ch}\left(F^{[n]}\right)=\frac{1}{n} \mathfrak{q}_{1}\left(1_{S}\right) \operatorname{ch}\left(F^{[n-1]}\right)+\sum_{\nu=0}^{2 n} \frac{\mathfrak{q}_{1}^{(\nu)}(\operatorname{ch}(F))}{\nu !} \frac{\mathfrak{q}_{1}\left(1_{S}\right)^{n-1}}{(n-1) !}|0\rangle .
$$

Computations with MAude [4] (see Part IV) give the following values (for each value, we extract the factor $1 /(\lambda ! \cdot|\lambda| !))$.

\begin{tabular}{c|cccccc}
\hline$\lambda$ & $(1)$ & $(1,1)$ & $(2)$ & $(1,1,1)$ & $(2,1)$ & $(3)$ \\
\hline$\gamma$ & 0 & $-\frac{1}{4} \cdot \frac{1}{3}$ & $-\frac{1}{2} \cdot 1$ & $\frac{1}{36} \cdot \frac{3}{5}$ & $\frac{1}{6} \cdot \frac{3}{4}$ & $\frac{1}{6} \cdot 1$ \\
$\delta$ & 0 & $-\frac{1}{4} \cdot \frac{1}{12}$ & $-\frac{1}{2} \cdot \frac{1}{6}$ & $\frac{1}{36} \cdot \frac{7}{30}$ & $\frac{1}{6} \cdot \frac{7}{20}$ & $\frac{1}{6} \cdot \frac{7}{12}$ \\
\hline
\end{tabular}

\begin{tabular}{c|ccccc}
\hline$\lambda$ & $(1,1,1,1)$ & $(2,1,1)$ & $(2,2)$ & $(3,1)$ & $(4)$ \\
\hline$\gamma$ & $-\frac{1}{576} \cdot \frac{29}{35}$ & $-\frac{1}{48} \cdot \frac{29}{30}$ & $-\frac{1}{48} \cdot \frac{11}{10}$ & $-\frac{1}{24} \cdot \frac{6}{5}$ & $-\frac{1}{24} \cdot \frac{3}{2}$ \\
$\delta$ & $-\frac{1}{576} \cdot \frac{59}{140}$ & $-\frac{1}{48} \cdot \frac{59}{105}$ & $-\frac{1}{48} \cdot \frac{43}{60}$ & $-\frac{1}{24} \cdot \frac{5}{6}$ & $-\frac{1}{24} \cdot \frac{5}{4}$ \\
\hline
\end{tabular}

REMARK 4.2. This computation shows in particular that the functions $g_{1}$ and $g_{2}$ are not integer-valued and one may suppose that they are always negative.

\section{The Chern class}

\subsection{The rank 1 case}

In the case of a vector bundle $F$ of rank 1 , there is a complete answer to the question of the determination of the universal constants for the Chern class.

Formula 5.1 (Lehn [6, Theorem 4.6]). Let L be a line bundle on $S$. The generating series of the Chern classes of tautological bundles takes the form:

$$
\mathrm{C}(L):=\mathfrak{c}(L)|1\rangle=\exp \left(\sum_{k \geqslant 1} \frac{(-1)^{k-1}}{k} \mathfrak{q}_{k}(\mathrm{c}(L))\right)|0\rangle .
$$

In particular, for $\lambda=(k), u_{\lambda}^{\mathrm{c}}=\left((-1)^{k-1} / k\right) \mathrm{c}(L)+(r-1)(\cdots)$ and $u_{\lambda}^{\mathrm{c}}=$ $(r-1)(\cdots)$ otherwise.

In this formula, one sees that the only operators $\mathfrak{q}_{\lambda}$ occurring have partitions with one part, and that the invariants $K_{S}$ and $e_{S}$ do not appear. It is not expected that this will remain true - neither for other characteristic classes, nor in higher rank. There are no similar formulas known for other multiplicative characteristic classes.

\subsection{Bundles of higher rank}

For a bundle $F$ of rank $r \geqslant 1$, information on the beginning of the universal formula of the Chern class is contained in the following result, obtained by specialization to the affine plane. 
Formula 5.2 (Boissière \& Nieper-Wißkirchen [3, Theorem 4]). Let $F$ be the trivial bundle of rank $r$ on $\mathbb{C}^{2}$. Then:

$$
\mathrm{C}(F)=\exp \left(\sum_{k \geqslant 1} \frac{(-1)^{k-1}}{k^{2}}\left(\begin{array}{c}
r \cdot k \\
k-1
\end{array}\right) \mathfrak{q}_{k}\left(1_{\mathbb{C}^{2}}\right)\right)|0\rangle .
$$

In particular, this gives the complete answer for the degree zero part of the $u_{(k)}$. In order to get more information, we implement the commutation relation of Lehn [6, Theorem 4.2]:

$$
\mathfrak{c}(F) \circ \mathfrak{q}_{1}\left(1_{S}\right) \circ \mathfrak{c}(F)^{-1}=\sum_{\nu, k \geqslant 0}\left(\begin{array}{c}
\operatorname{rk}(F)-k \\
\nu
\end{array}\right) \mathfrak{q}_{1}^{(\nu)}\left(c_{k}(F)\right),
$$

which gives the following recursion formula:

$$
c\left(F^{[n]}\right)=\frac{1}{n}\left(\sum_{\substack{0 \leqslant k \leqslant \operatorname{rk}(F) \\
0 \leqslant \nu \leqslant \operatorname{rk}(F)-k}}\left(\begin{array}{c}
\operatorname{rk}(F)-k \\
\nu
\end{array}\right) \mathfrak{q}_{1}^{(\nu)}\left(c_{k}(F)\right)\right) \mathrm{c}\left(F^{[n-1]}\right) .
$$

Computations with MAUdE [4] give the series inside the exponential. For example, in the rank 2 case the first $u_{\lambda}^{\mathrm{c}}$ computed in the basis $1, c_{1}, K, c_{2}, c_{1}^{2}, c_{1} K, K^{2}, e$ are as follows.

\begin{tabular}{c|c}
\hline$\lambda$ & $u_{\lambda}^{\mathrm{c}}$ \\
\hline$(1)$ & $1+c_{1}+c_{2}$ \\
\hline$(1,1)$ & $-\frac{1}{2}\left(1+c_{1}+c_{2}\right)$ \\
$(2)$ & $-\left(1+\frac{1}{2}\left(3 c_{1}+K+2 c_{2}+c_{1}^{2}+c_{1} K\right)\right)$ \\
\hline$(1,1,1)$ & $\frac{1}{3}\left(1+c_{1}+c_{2}\right)$ \\
$(2,1)$ & $2+3 c_{1}+K+2 c_{2}+c_{1}^{2}+c_{1} K$ \\
$(3)$ & $\frac{5}{3}+\frac{10}{3} c_{1}+2 K+2 c_{2}+2 c_{1}^{2}+3 c_{1} K+\frac{2}{3} K^{2}-\frac{1}{3} e$ \\
\hline$(1,1,1,1)$ & $-\frac{1}{4}\left(1+c_{1}+c_{2}\right)$ \\
$(2,1,1)$ & $-\left(3+\frac{1}{2}\left(9 c_{1}+3 K+6 c_{2}+3 c_{1}^{2}+3 c_{1} K\right)\right)$ \\
$(2,2)$ & $-\frac{1}{4}\left(9+18 c_{1}+10 K+10 c_{2}+11 c_{1}^{2}+15 c_{1} K+3 K^{2}-e\right)$ \\
$(3,1)$ & $-\left(5+10 c_{1}+6 K+6 c_{2}+6 c_{1}^{2}+9 c_{1} K+2 K^{2}-e\right)$ \\
$(4)$ & $-\frac{1}{4}\left(14+35 c_{1}+29 K+20 c_{2}+30 c_{1}^{2}+58 c_{1} K+22 K^{2}-10 e\right)$ \\
\hline
\end{tabular}

REMARK 5.3. In order to recover Formula 5.2 for $S=\mathbb{C}^{2}$, set $c_{1}=0, c_{2}=0$, $K=0$ and $e=0$, and keep only the partitions with one part (since the diagonal push-forward is trivial in this case).

We can simplify the combinatorial difficulties by making the following assumptions: $S$ is an abelian surface and $F$ is a trivial bundle of rank $r$ over $S$. Concretely, in our computer program (Part IV) we set $c_{1}=0, c_{2}=0, e=0$ and $K=0$. Thus the elements $u_{\lambda}^{\mathrm{c}}$ inside the exponential restrict to their degree zero term $\left(u_{\lambda}^{\mathrm{c}}\right)_{0}$ for each partition. We get the following results. 
- Rank 2:

\begin{tabular}{c|cccccc}
\hline$\lambda$ & $(1)$ & $(1,1)$ & $(2)$ & $(1,1,1)$ & $(2,1)$ & $(3)$ \\
\hline$\left(u_{\lambda}^{\mathrm{c}}\right)_{0}$ & 1 & $-\frac{1}{2}$ & -1 & $\frac{1}{3}$ & 2 & $\frac{5}{3}$ \\
\hline
\end{tabular}

\begin{tabular}{c|ccccc}
\hline$\lambda$ & $(1,1,1,1)$ & $(2,1,1)$ & $(2,2)$ & $(3,1)$ & $(4)$ \\
\hline$\left(u_{\lambda}^{\mathrm{c}}\right)_{0}$ & $-\frac{1}{4}$ & -3 & $-\frac{9}{4}$ & -5 & $-\frac{7}{2}$ \\
\hline
\end{tabular}

\begin{tabular}{c|ccccccc}
\hline$\lambda$ & $(1,1,1,1,1)$ & $(2,1,1,1)$ & $(2,2,1)$ & $(3,1,1)$ & $(3,2)$ & $(4,1)$ & $(5)$ \\
\hline$\left(u_{\lambda}^{\mathrm{c}}\right)_{0}$ & $\frac{1}{5}$ & 4 & 9 & $\frac{61}{6}$ & 12 & 14 & $\frac{42}{5}$ \\
\hline
\end{tabular}

\begin{tabular}{|c|c|c|c|c|c|c|c|c|}
\hline$\lambda$ & $(1,1,1$ & $, 1,1,1)$ & $(2,1,1$ & $1,1)$ & $(2,2,1,1)$ & $(2,2,2)$ & $(3,1,1,1)$ & $(3,2,1)$ \\
\hline$\left(u_{\lambda}^{\mathrm{c}}\right)_{0}$ & & $-\frac{1}{6}$ & - & & $-\frac{2693}{120}$ & -9 & $-\frac{1007}{60}$ & $-\frac{907}{15}$ \\
\hline$\lambda$ & $(3,3)$ & $(4,1,1)$ & $(4,2)$ & $(5,1)$ & (6) & & & \\
\hline$\left(u_{\lambda}^{\mathrm{c}}\right)_{0}$ & $-\frac{50}{3}$ & $-\frac{2129}{60}$ & -35 & -42 & -22 & & & \\
\hline
\end{tabular}

- Rank 3:

\begin{tabular}{c|cccccc}
\hline$\lambda$ & $(1)$ & $(1,1)$ & $(2)$ & $(1,1,1)$ & $(2,1)$ & $(3)$ \\
\hline$\left(u_{\lambda}^{\mathrm{c}}\right)_{0}$ & 1 & $-\frac{3}{2}$ & $-\frac{3}{2}$ & 4 & 10 & 4 \\
\hline \multicolumn{7}{|c}{} \\
\hline$\lambda$ & $(1,1,1,1)$ & $(2,1,1)$ & $(2,2)$ & $(3,1)$ & $(4)$ \\
\hline$\left(u_{\lambda}^{\mathrm{c}}\right)_{0}$ & $-\frac{111}{8}$ & $-\frac{243}{4}$ & $-\frac{75}{4}$ & -42 & $-\frac{55}{4}$ \\
\hline
\end{tabular}

\begin{tabular}{c|ccccccc}
\hline$\lambda$ & $(1,1,1,1,1)$ & $(2,1,1,1)$ & $(2,2,1)$ & $(3,1,1)$ & $(3,2)$ & $(4,1)$ & $(5)$ \\
\hline$\left(u_{\lambda}^{\mathrm{c}}\right)_{0}$ & $\frac{553}{10}$ & $\frac{3553}{10}$ & $\frac{6051}{20}$ & $\frac{693}{2}$ & 168 & 198 & $\frac{273}{5}$ \\
\hline
\end{tabular}

- Rank 4:

\begin{tabular}{c|cccccc}
\hline$\lambda$ & $(1)$ & $(1,1)$ & $(2)$ & $(1,1,1)$ & $(2,1)$ & $(3)$ \\
\hline$\left(u_{\lambda}^{\mathrm{c}}\right)_{0}$ & 1 & -3 & -2 & 18 & 28 & $\frac{22}{3}$ \\
\hline \multicolumn{7}{|c}{} \\
\hline$\lambda$ & $(1,1,1,1)$ & $(2,1,1)$ & $(2,2)$ & $(3,1)$ & $(4)$ \\
\hline$\left(u_{\lambda}^{\mathrm{c}}\right)_{0}$ & -145 & -379 & $-\frac{147}{2}$ & -165 & -35 \\
\hline
\end{tabular}

- Rank 5:

\begin{tabular}{c|cccccc}
\hline$\lambda$ & $(1)$ & $(1,1)$ & $(2)$ & $(1,1,1)$ & $(2,1)$ & $(3)$ \\
\hline$\left(u_{\lambda}^{\mathrm{c}}\right)_{0}$ & 1 & -5 & $-\frac{5}{2}$ & $\frac{160}{3}$ & 60 & $\frac{35}{3}$ \\
\hline
\end{tabular}




\section{REMARK 5.4.}

- One notes that the signs are alternate: each partition $\lambda$ comes with a sign $(-1)^{|\lambda|-1}$.

- Looking at the partitions $(k)$, one recovers the coefficient $\frac{(-1)^{k-1}}{k^{2}}\left(\begin{array}{c}r \cdot k \\ k-1\end{array}\right)$ obtained in Formula (5.2).

- The coefficient for a partition $(k, 1)$ for $k \geqslant 2$, seems to be $\frac{(-1)^{k}(r-1)}{k+1}\left(\begin{array}{c}r \cdot k \\ k\end{array}\right)$.

- In the rank 2 case, it seems that $u_{\left(1^{k}\right)}^{\mathrm{c}}=\frac{(-1)^{k-1}}{k}\left(1+c_{1}+c_{2}\right)$ for $k \geqslant 1$.

- For partitions of length two, the results are compatible with those of [10].

\section{Other multiplicative characteristic classes}

The result of Proposition 5.2 generalizes to all multiplicative characteristic classes. By the splitting principle, any multiplicative characteristic class is uniquely determined by its value on line bundles, that is, by a power series $\phi(x) \in 1+x \mathbb{Q}[[x]]$. Define from $\phi$ a new power series $\psi(t)=\sum_{k \geqslant 1} \psi_{k} t^{k} \in t \mathbb{Q}[[t]]$ by the relation:

$$
\frac{\partial \psi}{\partial t}\left(\frac{x}{\phi(-x)}\right)=\phi(-x) \text {. }
$$

Then we have our next formula.

Formula 6.1 (Boissière \& Nieper-Wißkirchen [3, Theorem 4]). Let $F$ be the trivial bundle of rank $r$ on $\mathbb{C}^{2}$. Then:

$$
\Phi(F)=\exp \left(\sum_{k \geqslant 1} \frac{\psi_{k}}{k} \mathfrak{q}_{k}\left(1_{\mathbb{C}^{2}}\right)\right)|0\rangle
$$

\section{Part III. Characteristic Classes of the tangent Bundle}

In this part, we study the characteristic classes of the tangent bundle on $S^{[n]}$. We first recall the theoretical results. The study of the tangent bundle is related to the study of the tautological bundles: the latter are needed, for example, in the recursive computation of the Chern character (see Section 10.2).

\section{Shape of the formulas}

The first result concerns multiplicative characteristic classes.

TheOREm 7.1 (Boissière [1], Boissière \& Nieper-Wißkirchen [3]). Let $\phi$ be a multiplicative characteristic class. There are unique rational constants $a_{\lambda}, b_{\lambda}, c_{\lambda}$ and $d_{\lambda}$ such that the generating series of the $\phi$-classes of the tangent bundle on $S^{[n]}$ is given by:

$$
\Phi(S):=\exp \left(\sum_{\lambda \in \mathcal{P}} a_{\lambda} \mathfrak{q}_{\lambda}\left(1_{S}\right)+b_{\lambda} \mathfrak{q}_{\lambda}\left(e_{S}\right)+c_{\lambda} \mathfrak{q}_{\lambda}\left(K_{S}\right)+d_{\lambda} \mathfrak{q}_{\lambda}\left(K_{S}^{2}\right)\right)|0\rangle
$$


The case of the Chern character is particular but the structure is similar.

THEOREM 7.2 (Boissière [1]). There are unique rational constants $\alpha_{\lambda}, \beta_{\lambda}, \gamma_{\lambda}, \delta_{\lambda}$ such that the Chern character of the tangent bundle on $\operatorname{Hilb}(S)$ is given by:

$$
\operatorname{Ch}(S):=\left(\sum_{\lambda \in \mathcal{P}} \alpha_{\lambda} \mathfrak{q}_{\lambda}\left(1_{S}\right)+\beta_{\lambda} \mathfrak{q}_{\lambda}\left(e_{S}\right)+\gamma_{\lambda} \mathfrak{q}_{\lambda}\left(K_{S}\right)+\delta_{\lambda} \mathfrak{q}_{\lambda}\left(K_{S}^{2}\right)\right)|1\rangle .
$$

\section{The Chern class}

As a first step in the determination of the constants for multiplicative characteristic classes, we consider the special case of the Chern class. We wish to derive the constants in the universal formula:

$$
\mathrm{C}(S)=\exp \left(\sum_{\lambda \in \mathcal{P}} a_{\lambda} \mathfrak{q}_{\lambda}\left(1_{S}\right)+b_{\lambda} \mathfrak{q}_{\lambda}\left(e_{S}\right)+c_{\lambda} \mathfrak{q}_{\lambda}\left(K_{S}\right)+d_{\lambda} \mathfrak{q}_{\lambda}\left(K_{S}^{2}\right)\right)|0\rangle
$$

\subsection{Towards the $\left(1_{S}\right)$-series}

Proposition 8.1. For $k \geqslant 0$ we have:

$$
a_{2 k+2}=0, \quad a_{2 k+1}=\frac{(-1)^{k} C_{k}}{2 k+1},
$$

where $C_{k}:=(1 /(k+1))\left(\begin{array}{c}2 k \\ k\end{array}\right)$ is the kth Catalan number.

Proof. This result is proved in Boissière [1, Theorem 1.1]. We briefly recall the main argument. Assume that the surface is the affine plane: $S=\mathbb{C}^{2}$. Then the formula for the Chern class is easier: the canonical class and the Euler class are zero, and all operators $\mathfrak{q}_{\lambda}$ for a partition $\lambda$ of length $\ell(\lambda)>1$ are also zero. So the sum in the exponential involves only the $1_{S}$-series, and in this series only the partitions of length one. This gives all coefficients $a_{\lambda}$ for $\lambda=(k), k \geqslant 1$, since the Chern class takes the form:

$$
\mathrm{C}\left(\mathbb{C}^{2}\right)=\exp \left(\sum_{k \geqslant 1} a_{k} \mathfrak{q}_{k}\left(1_{S}\right)\right)|0\rangle .
$$

The computation is done as follows, by the use of the equivariant cohomology of the Hilbert scheme $\left(\mathbb{C}^{2}\right)^{[n]}$ for the natural action of the torus $\mathbb{C}^{*}$.

Formula 8.2 (Boissière [1]). The Chern class is:

$$
\mathrm{C}\left(\mathbb{C}^{2}\right)=\exp \left(\sum_{k \geqslant 0} \frac{(-1)^{k} C_{k}}{2 k+1} \mathfrak{q}_{2 k+1}\left(1_{S}\right)\right)|0\rangle,
$$

where $C_{k}:=(1 /(k+1))\left(\begin{array}{c}2 k \\ k\end{array}\right)$ is the kth Catalan number.

This gives the constants as announced. 


\subsection{The $\left(1^{k}\right)$-series}

We give here all constants $a_{\left(1^{k}\right)}, b_{\left(1^{k}\right)}, c_{\left(1^{k}\right)}$ and $d_{\left(1^{k}\right)}(k \geqslant 1)$ in Formula (1) of the Chern class. Firstly, an application of a result of Göttsche [5] gives the series $b, d$.

Proposition 8.3. For $k \geqslant 1$ we have:

1. $b_{\left(1^{k}\right)}=\frac{1}{k} \sum_{i \mid k} i=: \frac{1}{k} \sigma_{1}(k)$;

2. $d_{\left(1^{k}\right)}=0$.

Proof. 1. We specialize the general formula to the case when $S$ is a K3 surface. Since $K_{S}=0$, all terms involving the classes $K_{S}$ or $K_{S}^{2}$ disappear. The total Chern class takes the form:

$$
\mathrm{C}(S)=\exp \left(\sum_{\lambda \in \mathcal{P}} a_{\lambda} \mathfrak{q}_{\lambda}\left(1_{S}\right)+b_{\lambda} \mathfrak{q}_{\lambda}\left(e_{S}\right)\right)|0\rangle .
$$

Since the cohomological degree of an operator $\mathfrak{q}_{\lambda}(\alpha)$ is:

$$
\operatorname{deg} \mathfrak{q}_{\lambda}(\alpha)=2(|\lambda|+\ell(\lambda))+|\alpha|-4,
$$

the only way to get in conformal weight $n$ a class of maximal degree $4 n$ is to use the operators $\mathfrak{q}_{\left(1^{k}\right)}\left(e_{S}\right)$. This means that:

$$
\sum_{n \geqslant 0} e\left(S^{[n]}\right)=\exp \left(\sum_{k \geqslant 1} b_{\left(1^{k}\right)} \mathfrak{q}_{\left(1^{k}\right)}\left(e_{S}\right)\right)|0\rangle .
$$

Denote by $\chi_{S}$ the Euler characteristic of $S: \chi_{S}=\int_{S} e_{S}$ or equivalently $e_{S}=\chi_{S} x$ where $x$ denotes the cohomology class of a point. Since

$$
\Delta_{!}^{k} e_{S}=\frac{1}{\chi_{S}^{k-1}} e_{S} \otimes \cdots \otimes e_{S},
$$

we get $\mathfrak{q}_{\left(1^{k}\right)}\left(e_{S}\right)=\chi_{S} \mathfrak{q}_{1}(x)^{k}$ with $\int_{S[k]} \mathfrak{q}_{1}(x)^{k}|0\rangle=1$. This implies that:

$$
\sum_{n \geqslant 0} \int_{S[n]} e\left(S^{[n]}\right) t^{n}=\exp \left(\chi_{S} \sum_{k \geqslant 1} b_{\left(1^{k}\right)} t^{k}\right) \text {. }
$$

Now:

$$
\sum_{n \geqslant 0} \int_{S^{[n]}} e\left(S^{[n]}\right) t^{n}=\sum_{n \geqslant 0} \operatorname{dim} H^{*}\left(S^{[n]}\right) t^{n}
$$

and using Göttsche's formula [5] we get:

$$
\begin{aligned}
\sum_{n \geqslant 0} \int_{S[n]} e\left(S^{[n]}\right) t^{n} & =\prod_{m \geqslant 1}\left(\frac{1}{1-t^{m}}\right)^{\chi_{S}} \\
& =\exp \left(-\chi_{S} \sum_{m \geqslant 1} \ln \left(1-t^{m}\right)\right) .
\end{aligned}
$$

This gives the relation $\sum_{m \geqslant 1} \ln \left(1-t^{m}\right)=\sum_{k \geqslant 1} b_{\left(1^{k}\right)} t^{k}$, and hence the result holds 
2. We make no assumption on the surface $S$, so the formula contains all terms:

$$
\mathrm{C}(S)=\exp \left(\sum_{\lambda \in \mathcal{P}} a_{\lambda} \mathfrak{q}_{\lambda}\left(1_{S}\right)+b_{\lambda} \mathfrak{q}_{\lambda}\left(e_{S}\right)+c_{\lambda} \mathfrak{q}_{\lambda}\left(K_{S}\right)+d_{\lambda} \mathfrak{q}_{\lambda}\left(K_{S}^{2}\right)\right)|0\rangle
$$

Since the operators $\mathfrak{q}_{\lambda}\left(K_{S}^{2}\right)$ have the same cohomological degree as the operators $\mathfrak{q}_{\lambda}\left(e_{S}\right)$, the same argument as in the first assertion gives in this case:

$$
\sum_{n \geqslant 0} e\left(S^{[n]}\right)=\exp \left(\sum_{k \geqslant 1} b_{\left(1^{k}\right)} \mathfrak{q}_{\left(1^{k}\right)}\left(e_{S}\right)+d_{\left(1^{k}\right)} \mathfrak{q}_{\left(1^{k}\right)}\left(K_{S}^{2}\right)\right)|0\rangle
$$

but since we have already obtained that

$$
\sum_{n \geqslant 0} e\left(S^{[n]}\right)=\exp \left(\sum_{k \geqslant 1} b_{\left(1^{k}\right)} \mathfrak{q}_{\left(1^{k}\right)}\left(e_{S}\right)\right)|0\rangle
$$

the operators $\mathfrak{q}_{\left(1^{k}\right)}\left(K_{S}^{2}\right)$ cannot contribute to the Euler classes. This forces the vanishing $d_{\left(1^{k}\right)}=0$ for all $k \geqslant 1$.

To get the two series $a$ and $c$, we make use of a result of Ohmoto [11] (in fact, this method recovers the series $b$ and $d$ obtained in the preceding proposition, since the new argument uses a generalization of Göttsche's formula).

Proposition 8.4. For $k \geqslant 1$ we have:

$$
a_{\left(1^{k}\right)}=-c_{\left(1^{k}\right)}=\frac{1}{k} \sum_{i \mid k} i=\frac{1}{k} \sigma_{1}(k) .
$$

Proof. We follow Ohmoto [11, Remark 2.4]. Set $S^{(n)}:=S^{n} / \mathfrak{S}_{n}$, the quotient of $S^{n}$ by the permutation action of the symmetric group $\mathfrak{S}_{n}$. The Hilbert-Chow morphism

$$
\pi: S^{[n]} \rightarrow S^{(n)}
$$

is a crepant resolution of singularities. The composite morphism

$$
S \stackrel{\Delta^{n}}{\longrightarrow} S^{n} \rightarrow S^{(n)}
$$

induces a map $\Delta^{(n)}: H^{*}(S) \rightarrow H^{*}\left(S^{(n)}\right)$ (this is the Poincaré dual of Ohmoto's $\left.D^{n}\right)$. For dimensional reasons, the cohomological push-forward $f_{!}: H^{*}\left(S^{[n]}\right) \rightarrow$ $H^{*}\left(S^{(n)}\right)$ vanishes on classes containing at least one operator $\mathfrak{q}_{i}$ with $i \geqslant 2$, and by definition $f_{!} \mathfrak{q}_{\left(1^{n}\right)}(\alpha)|0\rangle=\Delta^{(n)} \alpha$ for $\alpha \in H^{*}(S)$.

The total cohomology space $\bigoplus_{n \geqslant 0} H^{*}\left(S^{(n)}\right)$ is equipped with a natural product

$$
\odot: H^{*}\left(S^{(k)}\right) \times H^{*}\left(S^{(l)}\right) \rightarrow H^{*}\left(S^{(k+l)}\right)
$$

(see $[\mathbf{1 1}, \S 3.1])$ such that:

$$
f_{!}\left(\mathfrak{q}_{\left(1^{\left.k_{1}\right)}\right.}\left(\alpha_{1}\right) \cdots \mathfrak{q}_{\left(1^{k_{r}}\right)}\left(\alpha_{r}\right)|0\rangle\right)=\Delta^{\left(k_{1}\right)}\left(\alpha_{1}\right) \odot \cdots \odot \Delta^{\left(k_{r}\right)}\left(\alpha_{r}\right) .
$$

This gives us the image of the generating series of Formula (1):

$$
\begin{aligned}
& f_{!} \mathrm{C}(S) \\
& \quad=\exp \left(\sum_{k \geqslant 1} a_{\left(1^{k}\right)} \Delta^{(k)}\left(1_{S}\right)+b_{\left(1^{k}\right)} \Delta^{(k)}\left(e_{S}\right)+c_{\left(1^{k}\right)} \Delta^{(k)}\left(K_{S}\right)+d_{\left(1^{k}\right)} \Delta^{(k)}\left(K_{S}^{2}\right)\right)
\end{aligned}
$$

where the exponential has to be taken for the $\odot$-ring structure. 
Now we apply Ohmoto's formula [11, Formula (3)]:

$$
\begin{aligned}
f_{!} \mathrm{C}(S) & =\prod_{i \geqslant 1}\left(1-\Delta^{(i)}\right)^{-\mathrm{c}(S)} \\
& =\prod_{i \geqslant 1} \exp \left(-\ln \left(1-\Delta^{(i)}\right)(\mathrm{c}(S))\right. \\
& =\exp \left(\sum_{i \geqslant 1} \sum_{j \geqslant 1} \frac{1}{j} \Delta^{(i \cdot j)}\left(1_{S}-K_{S}+e_{S}\right)\right) \\
& =\exp \left(\sum_{k \geqslant 1} \sigma_{1}(k)\left(\Delta^{(k)}\left(1_{S}\right)+\Delta^{(k)}\left(e_{S}\right)-\Delta^{(k)}\left(K_{S}\right)\right)\right) .
\end{aligned}
$$

This gives $a_{\left(1^{k}\right)}=b_{\left(1^{k}\right)}=\sigma_{1}(k), c_{\left(1^{k}\right)}=-\sigma_{1}(k)$ and $d_{\left(1^{k}\right)}=0$.

\section{9. $\quad$ Other multiplicative classes}

Generalizing the Chern class to a multiplicative class $\phi$ gives other series of universal coefficients:

$$
\Phi(S)=\exp \left(\sum_{\lambda \in \mathcal{P}} a_{\lambda} \mathfrak{q}_{\lambda}\left(1_{S}\right)+b_{\lambda} \mathfrak{q}_{\lambda}\left(e_{S}\right)+c_{\lambda} \mathfrak{q}_{\lambda}\left(K_{S}\right)+d_{\lambda} \mathfrak{q}_{\lambda}\left(K_{S}^{2}\right)\right)|0\rangle .
$$

\section{1. $\quad$ Towards the $\left(1_{S}\right)$-series}

We proceed as in the Chern class case. For $S=\mathbb{C}^{2}$, the $\phi$-class takes the form:

$$
\Phi\left(\mathbb{C}^{2}\right)=\exp \left(\sum_{k \geqslant 1} a_{k} \mathfrak{q}_{k}\left(1_{S}\right)\right)|0\rangle .
$$

This computation has been done in Boissière \& Nieper-Wißkirchen [3]. By the splitting principle, any characteristic class is uniquely determined by its value on a line bundle, that is, by a power series $\phi(x) \in 1+x A[[x]]$. Define from $\phi$ a new power series $\psi(t)=\sum_{k \geqslant 1} \psi_{k} t^{k} \in t A[[t]]$ by the relation:

$$
\frac{\partial \psi}{\partial t}\left(\frac{x}{\phi(x) \phi(-x)}\right)=\phi(x) \phi(-x) .
$$

Then we have the following formula.

Formula 9.1 (Boissière \& Nieper-Wißkirchen [3, Theorem 4]).

$$
\Phi\left(\mathbb{C}^{2}\right)=\exp \left(\sum_{k \geqslant 1} \frac{\psi_{k}}{k} \mathfrak{q}_{k}\left(1_{S}\right)\right)|0\rangle .
$$

REMARK 9.2. The series $\psi$ is odd, so all coefficients $\psi_{2 k}$ are zero.

This general formula contains some nice special cases.

- For $\phi(x)=1+x$, one gets the Chern class (Formula 8.2). 
- For $\phi(x)=1 /(1+x)$, one gets the Segré class as in Formula 9.3.

Formula 9.3 (Boissière \& Nieper-Wißkirchen [3, Example 6]).

$$
\mathrm{S}\left(\mathbb{C}^{2}\right)=\exp \left(\sum_{k \geqslant 0} \frac{1}{(2 k+1)^{2}}\left(\begin{array}{c}
3 k \\
k
\end{array}\right) \mathfrak{q}_{2 k+1}\left(1_{S}\right)\right)|0\rangle
$$

- For $\phi(x)=\sqrt{x /(1-\exp (-x))}$ one gets the square root of the Todd class as in Formula 9.4.

Formula 9.4 (Boissière \& Nieper-Wißkirchen [3, Example 7]).

$$
(\sqrt{\mathrm{Td}})\left(\mathbb{C}^{2}\right)=\exp \left(\sum_{k \geqslant 0} \frac{1}{4^{k} \cdot(2 k+1) \cdot(2 k+1) !} \mathfrak{q}_{2 k+1}\left(1_{S}\right)\right)|0\rangle .
$$

\section{The Chern character}

We now consider the formula for the Chern character:

$$
\operatorname{Ch}(S)=\left(\sum_{\lambda \in \mathcal{P}} \alpha_{\lambda} \mathfrak{q}_{\lambda}\left(1_{S}\right)+\beta_{\lambda} \mathfrak{q}_{\lambda}\left(e_{S}\right)+\gamma_{\lambda} \mathfrak{q}_{\lambda}\left(K_{S}\right)+\delta_{\lambda} \mathfrak{q}_{\lambda}\left(K_{S}^{2}\right)\right)|1\rangle
$$

\subsection{Towards the $\left(1_{S}\right)$-series}

Proposition 10.1. For $k \geqslant 0$ we have:

$$
\alpha_{2 k+2}=0, \quad \alpha_{2 k+1}=\frac{2}{(2 k+1) !} .
$$

Proof. This is proved in Boissière [1, Theorem 1.1]. The argument is similar to the case of the Chern class (see Proposition 8.1), and the result is contained in the following formula.

Formula 10.2 (Boissière [1, Theorem 1.1]).

$$
\operatorname{Ch}\left(\mathbb{C}^{2}\right)=\left(\sum_{k \geqslant 0} \frac{2}{(2 k+1) !} \mathfrak{q}_{2 k+1}\left(1_{S}\right)\right)|1\rangle .
$$

This gives the constants as announced.

\subsection{Towards the complete series}

Denote by $\mathfrak{c h} T \in \operatorname{End}\left(\mathbb{H}_{S}\right)$ the operator acting by multiplication by $\operatorname{ch}\left(T_{S}^{n}\right)$ on each component of conformal weight $n$. In order to get information on the series, 
we proceed to an implementation of the recursive formula of Boissière [1, Lemma $3.12]^{1,2}$ :

$$
\begin{aligned}
{\left[\mathfrak{c h} T, \mathfrak{q}_{1}\left(1_{S}\right)\right]=} & \sum_{\nu} \frac{1}{\nu !} \mathfrak{q}_{1}^{(\nu)}\left(1_{S}\right) \\
& -\sum_{\nu} \frac{1}{\nu !}\left(\mathfrak{q}_{1}^{(\nu)} \circ \mathfrak{G}^{\vee}\right) \Delta_{!}^{2}\left(\operatorname{td}(S)^{-1}\right) \\
& +\sum_{\nu} \frac{(-1)^{\nu}}{\nu !} \mathfrak{q}_{1}^{(\nu)}\left(\exp \left(-K_{S}\right)\right) \\
& -\sum_{\nu} \frac{(-1)^{\nu}}{\nu !}\left(\mathfrak{q}_{1}^{(\nu)} \circ \mathfrak{G}\right) \Delta_{!}^{2}\left(\exp \left(-K_{S}\right) \operatorname{td}(S)^{-1}\right) \\
& -\mathfrak{q}_{1}\left(e_{S}\right)
\end{aligned}
$$

with

$$
\operatorname{td}(S)^{-1}=1+\frac{K_{S}}{2}+\frac{2 K_{S}^{2}-e_{S}}{12}
$$

For the implementation, the computation with the operators $\mathfrak{G}$ is explained in Section 4.2, and the case of the operators $\mathfrak{G}^{\vee}$ is similar, since it is easy to deduce from the results on $\mathfrak{G}$ the following commutation relation:

$$
\left[\mathfrak{G}^{\vee}(\alpha), \mathfrak{q}_{1}\left(1_{S}\right)\right]=\exp (-\operatorname{ad} \mathfrak{d})\left(\mathfrak{q}_{1}(\alpha)\right)
$$

This yields the following recursive formula:

$$
\begin{aligned}
\operatorname{ch}\left(T_{n}^{S}\right)=\frac{1}{n} \mathfrak{q}_{1}\left(1_{S}\right) \operatorname{ch}( & \left.T_{n-1}^{S}\right)-\frac{1}{n !} \mathfrak{q}_{1}\left(e_{S}\right) \mathfrak{q}_{1}\left(1_{S}\right)^{n-1}|0\rangle \\
+\frac{1}{n !} \sum_{\nu=0}^{2 n} \frac{1}{\nu !}\left(\mathfrak{q}_{1}^{(\nu)}\left(1_{S}\right) \mathfrak{q}_{1}\left(1_{S}\right)^{n-1}\right. & \\
& \quad\left(\mathfrak{q}_{1}^{(\nu)} \circ \mathfrak{G}^{\vee}\right) \Delta_{!}^{2}\left(1_{S}+\frac{K_{S}}{2}+\frac{2 K_{S}^{2}-e_{S}}{12}\right) \mathfrak{q}_{1}\left(1_{S}\right)^{n-1} \\
& +(-1)^{\nu} \mathfrak{q}_{1}^{(\nu)}\left(1_{S}-K_{S}+\frac{K_{S}^{2}}{2}\right) \mathfrak{q}_{1}(1)^{n-1} \\
& \left.\quad-(-1)^{\nu}\left(\mathfrak{q}_{1}^{(\nu)} \circ \mathfrak{G}\right) \Delta_{!}^{2}\left(1_{S}-\frac{K_{S}}{2}+\frac{2 K_{S}^{2}-e_{S}}{12}\right) \mathfrak{q}_{1}\left(1_{S}\right)^{n-1}\right)|0\rangle .
\end{aligned}
$$

${ }^{1}$ In the proof of $\left[\mathbf{1}\right.$, Proposition 3.10], the assumption that $\int_{S} b_{i} b_{j} \operatorname{td}(S)=\delta_{i, j}$ should be made only for cohomology with complex coefficients; otherwise one should write $\int_{S} b_{i} b_{j} \operatorname{td}(S)=\kappa_{i} \delta_{i, j}$ for some $\kappa_{i} \in \mathbb{Q}$. This does not affect the proof (just add the $\kappa_{i} \mathrm{~s}$ ) since

$$
\left.\Delta_{!}^{2}(\operatorname{td}(S))^{-1}\right)=\operatorname{ch}\left(\mathcal{O}_{\Xi^{1}}\right)=\operatorname{ch}\left(\mathcal{O}_{\Delta}\right)
$$

Note that there is an inaccuracy in the text since $\operatorname{td}(S)$ should be $\operatorname{td}(S)^{-1}$ at the end of the proof. ${ }^{2}$ There is a typo in a computation on [1, p. 776]: $\operatorname{ch}\left(\mathcal{O}_{S}-T_{S}+\omega_{S}^{\vee}\right)=e_{S}$. 
One gets the following series inside the brackets of Formula (2).

\begin{tabular}{c|cccccc}
\hline$\lambda$ & $(1)$ & $(1,1)$ & $(2)$ & $(1,1,1)$ & $(2,1)$ & $(3)$ \\
\hline$\alpha$ & 2 & $-\frac{3}{2}$ & 0 & $\frac{5}{9}$ & 0 & $\frac{1}{3}$ \\
$\beta$ & -1 & $\frac{5}{8}$ & 0 & $-\frac{7}{27}$ & 0 & $-\frac{5}{36}$ \\
$\gamma$ & -1 & $-\frac{5}{12}$ & -1 & $-\frac{1}{36}$ & $\frac{13}{12}$ & $-\frac{1}{6}$ \\
$\delta$ & $\frac{1}{2}$ & $\frac{1}{4}$ & $\frac{1}{2}$ & $\frac{53}{270}$ & $-\frac{5}{24}$ & $\frac{4}{9}$ \\
\hline
\end{tabular}

REMARK 10.3. The zeros are no surprise. In fact, for each partition $\lambda$ such that $|\lambda|+\ell(\lambda)$ is odd, $\alpha_{\lambda}=\beta_{\lambda}=0$ since if $S$ is a non-compact symplectic surface, these terms would contribute to the even part of the Chern character, which is zero. The results are compatible with those of [10].

\section{Part IV. An implementation with Maude}

For documentation about MAUDE, see [4] or http://maude.cs.uiuc.edu/.

The program code can be found in Appendix A.

\section{Final remarks}

Although we implemented lots of classes, things remain to be done, in particular:

- write and implement recursions for the Segre classes of tautological bundles;

- write and implement a recursion for the Chern class of the tangent bundle;

- find satisfactory models for the general terms of the series obtained by symbolic computations. These models are still missing.

We decided not to implement further operators here. The methods are more or less straightforward generalization of Lehn's ideas in [6], but the formulas would be very long: the complexity occurs in the decomposition of the characteristic class of the tensor product of a vector bundle with a line bundle.

\section{Appendix A. The script}

This appendix contains the script referred to in the paper, and can be found at http://www.Ims.ac.uk/jcm/10/Ims2006-045/appendix-a.

\section{References}

1. Samuel Boissière, 'Chern classes of the tangent bundle on the Hilbert scheme of points on the affine plane', J. Algebraic Geom. 14 (2005) 761-787. 255, 256, 258, 259, 262, 263, 267, 268

2. SAmuel Boissière, 'On the McKay correspondences for the Hilbert scheme of points on the affine plane', Math. Ann. 334 (2006) 419-438. 
3. Samuel Boissière and Marc A. Nieper-Wisskirchen, 'Universal formulas for characteristic classes on the Hilbert schemes of points on surfaces', $J$. Algebra, to appear. 255, 256, 258, 260, 262, 266, 267

4. Manuel Clavel, Francisco Durán, Steven Eker, Patrick Lincoln, Narciso Martí-Oliet, José Meseguer and José F. Quesada, 'Maude: specification and programming in rewriting logic', Theoret. Comput. Sci. 285 (2002) 187-243. 255, 259, 260, 269

5. Lothar Göttsche, 'The Betti numbers of the Hilbert scheme of points on a smooth projective surface', Math. Ann. 286 (1990) 193-207. 264

6. MANFRED LeHn, 'Chern classes of tautological sheaves on Hilbert schemes of points on surfaces', Invent. Math. 136 (1999) 157-207. 255, 259, 260, 269

7. MANFRED LeHn, 'Lectures on Hilbert schemes', Algebraic structures and moduli spaces, CRM Proc. Lecture Notes 38 (Amer. Math. Soc., Providence, RI, 2004) 1-30. 255

8. Wei-Ping Li, Zhenbo Qin and Weiqiang Wang, 'Hilbert schemes and $\mathcal{W}$ algebras', Int. Math. Res. Not. (2002) 1427-1456. 258

9. Hiraku Nakajima, 'Heisenberg algebra and Hilbert schemes of points on projective surfaces', Ann. of Math. (2) 145 (1997) 379-388. 256

10. MARC NiePER-Wisskirchen, 'Equivariant cohomology, symmetric functions and the Hilbert scheme of points on the total space of the invertible sheaf $\mathcal{O}_{\mathbf{p}_{1}}(-2)$ over the projective line', arXiv:math.AG/0610834. 262, 269

11. Toru Oнмото, 'Generating functions of orbifold Chern classes I: symmetric products', arXiv:math.AG/0604583. 265, 266

Samuel Boissière sb@math.unice.fr

http://math.unice.fr/ sb

Laboratoire J. A. Dieudonné

UMR CNRS 6621

Université de Nice Sophia-Antipolis

Parc Valrose

06108 Nice

France

Marc A. Nieper-Wißkirchen nieper@mathematik.uni-mainz.de http://www . mathematik.uni-mainz.de/Members/nieper

Institut für Mathematik

Johannes-Gutenberg Universität

55099 Mainz

Germany 